Published online 2017 April 13.

Abstract

\title{
Periventricular Leucomalacia and Seizure in Preterm Infants
}

\author{
Mahtab Amirheidari, ${ }^{1,}{ }^{*}$ Reza Nafisi Moghadam, ${ }^{1}$ and Mona Kharraji ${ }^{1}$ \\ ${ }^{1}$ Shahid Sadoughi Yazd University of Medical Sciences, Yazd, Iran \\ "Corresponding author: Mahtab Amirheidari, Shahid Sadoughi Yazd University of Medical Sciences, Yazd, Iran. E-mail: amiheidarimahtab@gmail.com
}

Received 2016 December 21; Accepted 2017 February 08.

\begin{abstract}
Background: Prematurity neuropathology can be categorized as: periventricular leukomalacia (PVL), white matter changes, and basal ganglion, cortex and thalamus disorders. These abnormalities may induce seizure in preterm neonate. The aim of this study was to evaluate the association between white matter abnormalities and seizure in 18 months preterm infants with delay Neurodevelopment (ND).

Methods: This is an analytical cross-sectional study on 18 months preterm infants with ND delay who were referred to MRI department of Shaheed Sadoughi hospital of Yazd, 2015 - 2016. The information of birth age (weeks), gender, seizure, and PVL on MRI were collected. All statistical analysis was done by SPSS19.

Results: Totally 45 infants were studied. About 55.6\% were male and $44.6 \%$ female. The mean of pregnancy duration was 32.72 2.78 weeks. Seizure frequency was $42.2 \%$. The PVL grading was: $28.9 \%$ mild, $20 \%$ moderate and $4.4 \%$ sever. There was a statistically significance between PVL and birth age (week), Also between seizure and PVL.

Conclusions: The high light finding of our study was the higher frequency of seizure in infants without PVL. These findings can be explained by the inclusion criteria of our study.
\end{abstract}

Keywords: Periventricular Leukomalacia, Seizure, Preterm Infant

This is an abstract presented in the 33rd Iranian congress of radiology (ICR) and the 15th congress of Iranian radiographic science association (IRSA). 